\title{
Aprender brincando: validação semântica de tecnologia educacional sobre tuberculose para crianças escolares
}

\author{
Learning through play: semantic validation of educational technology on tuberculosis for school children \\ Aprender jugando: validación semántica de tecnología educativa sobre tuberculosis para niños \\ estudiantes
}

Ivaneide Leal Ataíde Rodrigues ${ }^{1}$ (B)

Laura Maria Vidal Nogueira ${ }^{1}$ (D)

Alexandre Aguiar Pereira² (D)

Paula Daniella de Abreu $^{3}$ (D)

Lucila Castanheira Nascimento ${ }^{4}$ (1)

Eliane Maria Ribeiro de Vasconcelos ${ }^{5}$ (B)

Marta Angélica lossi Silva ${ }^{4}$ (1)

Claudia Benedita dos Santos ${ }^{4}$ (1)

1. Universidade do Estado do Pará, Escola

de Enfermagem Magalhães Barata,

Departamento de Enfermagem Comunitária.

Belém, PA, Brasil.

2. Universidade do Estado do Pará, Escola de Enfermagem Magalhães Barata, Programa de Pós-Graduação em Enfermagem. Belém, PA, Brasil.

3. Universidade de São Paulo, Escola de Enfermagem de Ribeirão Preto, Programa de Pós-graduação de Enfermagem em Saúde Pública. Ribeirão Preto, SP, Brasil.

4. Universidade de São Paulo, Escola de Enfermagem de Ribeirão Preto, Departamento de Enfermagem Materno-Infantil e Saúde Pública. Ribeirão Preto, SP, Brasil.

5. Universidade Federal de Pernambuco, Departamento de Enfermagem em Saúde Pública. Recife, PE, Brasil.

Autor correspondente: Ivaneide Leal Ataíde Rodrigues. E-mail: ilar@globo.com

Recebido em 16/12/2020.

Aprovado em 17/03/2021.

DOI:https://doi.org/10.1590/2177-9465-EAN-2020-0492

\section{Resumo}

Objetivo: validar semanticamente tecnologia educacional sobre tuberculose para crianças escolares. Método: estudo metodológico, realizado com 51 escolares, entre 10 e 12 anos, de escola pública em Belém, Pará. Na coleta de dados, apresentou-se às crianças a tecnologia educacional, um caça-palavras, com definição, transmissão, sinais e sintomas da tuberculose e, após sua aplicação realizou-se entrevista individual, com perguntas abertas e fechadas, sobre atributos associados à impressão geral ao conteúdo e forma. Para descrever a concordância das respostas à entrevista, foi utilizado o índice de validade de conteúdo, com ponto de corte igual a 0,80 . Na descrição das variáveis numéricas, utilizou-se o software Statistical Package for the Social Sciences, 22.0 e, para o corpus textual, empregou-se o software IRaMuTeQ 0.7, alpha 2. Resultados: os índices de validade de conteúdo apresentaram resultados superiores a $90 \%$. A descrição dos depoimentos gerou duas nuvens de palavras, referentes à compreensão sobre a doença e capacidade informativa da tecnologia. Conclusão e implicações para a prática: a tecnologia foi considerada de fácil utilização, relevante e de conteúdo apropriado, portanto, válida para crianças compreenderem a doença possibilitando atuarem como multiplicadores da informação para sua rede de convívio.

Palavras-chave: Educação em Saúde; Tuberculose; Criança; Tecnologia Educacional; Estudos de Validação.

\section{Abstract}

Objective: to semantically validate tuberculosis educational technology for school children. Method: a methodological study, conducted with 51 students aged 10 to 12 years old, from a public school in Belém, Pará. For data collection, the children were presented with the educational technology, a word search, with definition, transmission, signs and symptoms of tuberculosis and, after its application, an individual interview was carried out, with open and closed questions about attributes associated with the general impression, content and form. To describe the agreement of the answers to the interview, the content validity index was used, with a cutoff point equal to 0.80 . To describe the numerical variables, we used the software Statistical Package for the Social Sciences, 22.0, and for the text corpus, we used the software IRaMuTeQ 0.7, alpha 2. Results: the content validity indexes presented results higher than $90 \%$. The description of the statements generated two word clouds, referring to the understanding about the disease and the informative capacity of the technology. Conclusion and implications for practice: the technology was considered easy to use, relevant and with appropriate content, therefore, valid for children to understand the disease, enabling them to act as multipliers of information to their social network.

Keywords: Health Education; Tuberculosis; Child; Educational Technology; Validation Studies.

\section{Resumen}

Objetivo: validar semánticamente la tecnología educativa sobre tuberculosis para niños estudiantes. Método: estudio metodológico realizado con 51 estudiantes, entre 10 y 12 años, de una escuela pública de Belém, Pará. En la recolección de datos, se presentó a los niños la tecnología educativa, un buscador de palabras, con definición, transmisión, signos y síntomas de tuberculosis y, luego de su aplicación, se realizó una entrevista individual, con preguntas abiertas y cerradas, sobre atributos asociados a la impresión general, contenido y forma. Para describir la concordancia de las respuestas a la entrevista se utilizó el índice de validez de contenido, con un punto de corte igual a 0,80. En la descripción de las variables numéricas se utilizó el software Statistical Package for the Social Sciences, 22.0 y, para el corpus textual, se utilizó el software IRaMuTeQ 0.7, alfa 2. Resultados los índices de validez de contenido presentaron resultados superiores al $90 \%$. La descripción de los testimonios generó dos nubes de palabras, referidas a la comprensión de la enfermedad y la capacidad de información de la tecnología. Conclusión e implicaciones para la práctica: la tecnología se consideró fácil de usar, relevante y con contenido adecuado, así que, válida para que los niños comprendan la enfermedad, posibilitándoles actuar como multiplicadores de información para su red social.

Palabras clave: Educación en Salud; Tuberculosis; Niño; Tecnología Educacional; Estudio de Validación. 


\section{INTRODUÇÃO}

A tuberculose é, mundialmente, um importante desafio à saúde coletiva. Segundo a Organização Mundial da Saúde (OMS), 95\% dos casos e mortes pela doença ocorrem em países em desenvolvimento, sendo considerada como a principal causa de morte por doenças infecciosas em todo o mundo. Países como o Brasil, Federação Russa, Índia, China e África do Sul, que compõem o BRICS, representam cerca de $50 \%$ dos casos de tuberculose no mundo. ${ }^{1}$

O cenário mundial levou a OMS a definir, para o período de 2016 a 2020, um novo elenco de países prioritários, nele estão três listas onde constam 30 países que, segundo suas características epidemiológicas, representam $87 \%$ dos casos da doença no mundo, sendo que o Brasil figura em duas dessas listas, ocupando a 20ª posição na classificação de carga da doença. Para o Ministério da Saúde (MS), desde 2003, a doença é considerada como prioritária na agenda política, com diagnóstico e tratamento disponíveis na rede do Sistema Único de Saúde (SUS). ${ }^{2}$

No Brasil, em 2019, foi registrado coeficiente de incidência de 35,0/100 mil habitantes e de mortalidade 2,2 óbitos/100 mil habitantes. No Pará, foram 4.885 casos notificados, com coeficiente de incidência de 55,8/100 mil habitantes. Entende-se que a incidência e o desfecho da tuberculose estão associados à determinação social do processo saúde-doença. A vulnerabilidade individual, social e programática, sobretudo no contexto de crise econômica do País, contribuiu com o aumento da incidência da doença nos subcenários. ${ }^{3}$

Apesar dos progressos para seu diagnóstico, tratamento e controle, a tuberculose permanece, contemporaneamente, um importante problema de saúde pública. Assim, é necessário o incremento de estratégias de educação em saúde no âmbito da Atenção Primária à Saúde (APS), com o objetivo de empoderar pessoas acerca do diagnóstico, prevenção e tratamento, para que elas sejam corresponsáveis pelo controle da cadeia de transmissibilidade, sobretudo em regiões de maior vulnerabilidade social. ${ }^{4}$

Nesse contexto, a escola torna-se um privilegiado espaço para práticas de promoção da saúde e prevenção de agravos, para o desenvolvimento de conhecimentos, habilidades e mudanças de comportamento, principalmente por reunir crianças e adolescentes em período de experimentação, maturação e múltiplas transições físicas, psicológicas e sociais. ${ }^{5}$

Assim, entende-se a necessidade de utilizar estratégias atrativas, críticas e inovadoras de educação em saúde, para que possam ampliar seus conhecimentos e que, empoderados, sejam agentes replicadores e executores de ações de promoção da saúde no seu cotidiano. ${ }^{6}$

A educação em saúde é um conjunto de práticas pedagógicas que viabiliza um processo de aprendizagem e ensino contínuo, dinâmico, complexo e planejado, que leva em conta os fatores internos e externos dos indivíduos, os quais influenciam seu estado de saúde e seu potencial de melhoria de conhecimentos, habilidades e atitudes em relação às suas necessidades e comportamentos no contexto da saúde. ${ }^{7}$

A utilização de tecnologias educacionais viabiliza a troca de saberes e pactua com a valorização do conhecimento prévio, somado ao conhecimento científico, para a problematização na construção conjunta do saber em saúde. Com isso, potencializa-se a possibilidade dos sujeitos se tornarem agentes de transformação, tanto no âmbito individual como no coletivo. ${ }^{8}$

Ao longo do desenvolvimento humano e do saber científico, o termo tecnologia foi atribuído à concepção de máquinas, bens físicos utilizáveis, bem como a procedimentos técnicos, em uma visão reducionista. Novos estudos permitiram desenvolver conhecimento sobre o tema, ampliando o conceito e demonstrando que podem ser entendidas como resultado da experiência cotidiana e de pesquisas, desenvolvendo conhecimentos científicos para criação de produtos materiais ou saberes que possibilitam a intervenção em situações práticas. ${ }^{9}$

A tecnologia educacional mostra-se como ferramenta apropriada para proporcionar aprendizado que possa tornar as crianças agentes do conhecimento, já que favorecem a participação dos sujeitos no processo educativo, contribuindo para a construção da cidadania e o aumento da autonomia dos envolvidos. ${ }^{10}$ Para que desempenhem esse papel, é necessário que passem por processo de validação para atribuir confiabilidade ao seu conteúdo. ${ }^{11}$ Dessa forma, este estudo teve como objetivo validar semanticamente tecnologia educacional sobre tuberculose para crianças escolares.

\section{MÉTODO}

Trata-se de uma pesquisa metodológica, que é um tipo de pesquisa que abrange a produção/construção, validação e avaliação de novos produtos. ${ }^{12}$ Estudo multicêntrico com colaboração internacional, desenvolvido como parte de um projeto de pesquisa em cooperação acadêmica, com participação de cinco Universidades Públicas e financiado por órgão governamental.

A tecnologia validada, neste estudo, resultou de projeto também desenvolvido no âmbito do estudo multicêntrico. Foi desenvolvida em duas etapas inter-relacionadas. Inicialmente, com realização de círculos de cultura para formação de adolescentes escolares multiplicadores em saúde, sob os pressupostos da educação multicultural e da promoção da saúde e a construção e apresentação da tecnologia. Considerou-se na construção documentos oficiais e publicações referentes à tuberculose e à educação em saúde com escolares. Em seguida, foi feita a avaliação da mesma por meio do método Delphi junto a especialistas em saúde e educação. ${ }^{13}$

Essa etapa de validação semântica foi desenvolvida em uma escola pública municipal, localizada em bairro periférico em Belém, Pará. Esse bairro foi selecionado como cenário da pesquisa por registrar, anualmente, expressivo número de casos de tuberculose, segundo dados da Secretaria Municipal de Saúde (SMS) de Belém, e corroborado pelos resultados de dissertação de mestrado, também vinculada ao projeto de pesquisa financiado por órgão governamental. ${ }^{14,15}$ 
A população de estudo constituiu-se de escolares brasileiros, na faixa etária de 10 a 12 anos e o número amostral foi definido segundo o Manual do software Interface de R pour les Analyses Multidimensionnelles de Textes et de Questionnaires (IRaMuTeQ), versão 0.7 , alpha $2 .{ }^{16}$ Foram incluídos escolares regularmente matriculados e com frequência atualizada, considerando-se o período de coleta de dados, que ocorreu no mês de fevereiro de 2019. O grupo de participantes foi selecionado por conveniência, pois foram convidadas a participar todas as crianças elegíveis que compareceram à escola nos dias e horários estabelecidos pela coordenação institucional para apresentação do projeto $e$ coleta de dados. Foram excluídas aquelas que não compareceram, após duas tentativas de convite no período de coleta de dados.
A coleta de dados ocorreu nas dependências da escola, em salas predeterminadas pela coordenação e foi dividida em dois momentos. No primeiro, foi apresentada aos estudantes, a tecnologia a ser validada, um jogo do tipo caça-palavras cujo objetivo é trabalhar a prevenção e o controle da tuberculose com escolares, abordando aspectos referentes à definição da doença (o que é tuberculose?), modos de transmissão (como passa de uma pessoa para outra?), os sinais e sintomas (o que pode ser observado em uma pessoa doente ou o que ela sente?), e onde procurar ajuda e tratamento (que serviços ou profissionais podem ser procurados em caso de adoecimento?).

A versão final do jogo (Figura 1) está apresentada em uma página impressa, contendo texto explicativo inicial com nove linhas,

\section{Caça Palavras}

\section{Tuberculose}

A tuberculose é uma doença causada pelo Mycobacterium tuberculosis, também conhecido por Bacilo de Kock. Étransmitida de uma pessoa para outra por meio da saliva que sai da boca do doente, quando ele fala, tosse ou espirra. Lugares fechados e com muitas pessoas juntas aumentam as chances de espalhar o bacilo outras pessoas serem contaminadas. Uma pessoa quando está com tuberculose pode apresentar: tosse com catarro, falta de apetite, perda de peso, febre baixa, muito suor e cansaço. Em casos graves, pode haver catario com sangue.

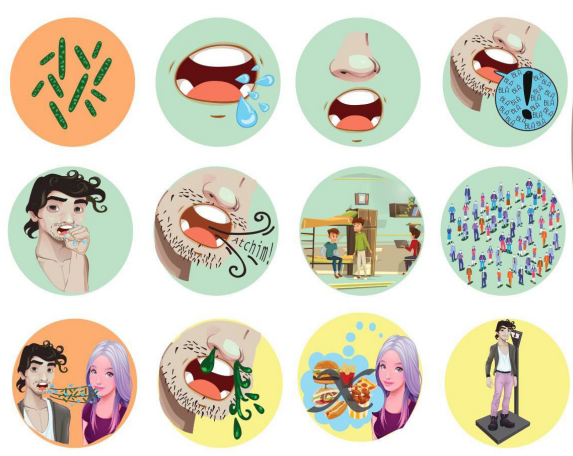

\section{Vamos ao trabalho: Procurem as palavnas}

$M F R R R R-V I C M R O V$ T $R$ L N C

Y A L T T A D D T A A P E T T I T E A D D O D I I D

C E S O I J U C B F V R O A J M T C S D

O P P S P E B B O C A A D O D O O E N T E O M

$B$ I S S E R E E C L R F C C N E O D C U

A E L E S A R F I A R A A S M I S S A B C T M A S A C A L U A M T C A J T R N M T I P L O M U C O B A C A E R I U M S U E T N M A M L A D E S P R R R A V T B A A R E A I F I O N E R O M R A F I E R C I I PRCRESA I I VAOLALEROD U A N A E N E A O U A J V S L U I I T E M D M P S T I C C L A T P A T G V R D L T I A D P P E R O K K O S R R E A A A I E F E U S T L A F A T S S B I S S D R B U I $B$ A C I L O D E K O C C K S R E E C O A B E E D D H C L S N F E C O O A S N T A C R M A B A H E P A V U F A C P F E B R $C I V I I R A B$ I S U O R S A E E A S I U N R C O O D O R R N T E B J I T C T D U L A D $\quad K$ K B O C C R O I I D VU/C I H L O E O C M M A F A A A R O C N P TA I V S U $S A R I C M D V R U M A T M E D U S F L$

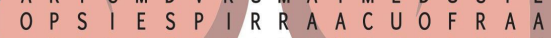
$S$ A D K L O C E O I D V S C U S L O E C I C M M O F A L A R O C A P A M I V S U A R I O M D V R U M A D M A C U S FL O OPS PER D A D E PESOSFRAA

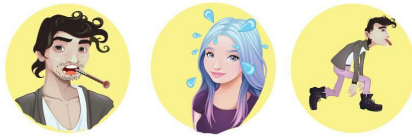

Trabalhando com as palainas encontradas:
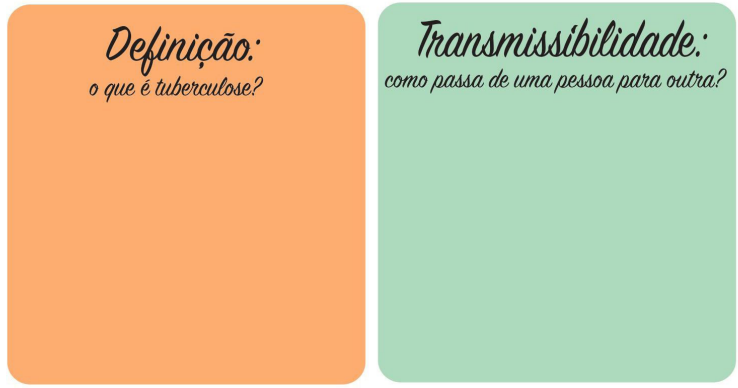

Sinais e sintomas. o que pode ser observado em uma pessoa com tuberculose?

Figura 1. Versão final do caça-palavras sobre tuberculose para crianças escolares. Belém, Pará, Brasil, 2019. Fonte: Elaborada pelos autores. 
que traz informações sobre a doença, ladeado pelo respectivo conjunto de palavras para realizar a busca. Neste, constam 15 palavras, extraídas do texto inicial, que devem ser identificadas pelos participantes. Apresenta, ainda, 15 figuras representativas das expressões descritas no texto e três quadros em branco, de cores diferentes, onde devem ser escritos a definição da doença, formas de transmissão e sinais e sintomas característicos, de acordo com as palavras encontradas.

Após a apresentação do caça-palavras, foi explicada a dinâmica do trabalho e feita a leitura do texto, esclarecendo seu conteúdo. Foram entregues exemplares a cada um dos participantes, solicitando que identificassem as palavras no conjunto de palavras do caça e descrevessem o que identificaram nos três quadros específicos, relacionando as palavras ao texto explicativo.

No segundo momento, realizou-se a entrevista individual, utilizando formulário contendo dez perguntas fechadas e duas abertas, elaborado pelos próprios autores, com base em um instrumento disponibilizado por um grupo europeu e adaptado para o Brasil por grupo de pesquisa cadastrado no Diretório do Conselho Nacional de Desenvolvimento Científico e Tecnológico $(\mathrm{CNPq}) .{ }^{17-19}$ As crianças responderam às questões sobre o caça-palavras, segundo um conjunto de três blocos, a saber: aparência geral, compreensão e relevância do texto e sobre seu formato.

As questões semiestruturadas versaram sobre a relevância da tecnologia expressas na compreensão das crianças, em relação às informações contidas no caça-palavras e sua aplicabilidade. Eram convidadas a descrever, com suas próprias palavras, o que entenderam das informações e sua aplicabilidade prática para outras pessoas conhecerem mais sobre a tuberculose. Essas questões foram gravadas em mídia digital ou registradas no próprio instrumento pelos pesquisadores, mediante consentimento dos responsáveis e das próprias crianças.

As questões fechadas consideraram os atributos referentes à forma e conteúdo que compuseram os três blocos de avaliação, considerando a impressão geral sobre o caça-palavras, o conteúdo do texto e formato da tecnologia, com as crianças respondendo em uma escala do tipo Likert de quatro itens, variando em um ranking onde 1 seria não relevante ou não representativo e 4 relevante ou representativo. ${ }^{11}$

Utilizou-se o Índice de Validade de Conteúdo (IVC) para a mensuração da concordância entre as respostas das crianças aos itens relacionados aos três blocos de avaliação do caça-palavras. O IVC foi calculado por meio da soma dos itens assinalados pelas crianças como relevantes/representativos, dividido pelo número total de itens considerados na avaliação. Levou-se em conta o ponto de corte igual a 0,80 para o IVC. ${ }^{11}$

Todos os momentos da coleta de dados foram conduzidos pela pesquisadora principal, auxiliada por sete membros do grupo de pesquisa, capacitados e supervisionados por ela. Foram realizadas reuniões prévias à coleta de dados para conhecerem a tecnologia, o instrumento de coleta de dados e a dinâmica das fases previstas para sua execução.
As informações numéricas foram organizadas em planilha eletrônica no Microsoft Excel, utilizando-se a técnica da dupla digitação para sua validação e descritos por meio do software Statistical Package for the Social Sciences (SPSS) versão 22.0, no de série 10101151049 .

O material textual foi organizado em um corpus e submetido ao processamento com auxílio do software IRaMuTeQ, de domínio público, e descrito segundo nuvem de palavras. Nesse tipo de análise lexical, as palavras são agrupadas por número de ocorrência e organizadas graficamente de acordo com a sua frequência no corpus submetido, permitindo facilmente sua identificação, bem como o processo de análise a ser feito pelos pesquisadores. ${ }^{16}$

O projeto atendeu à Resolução CNS/MS no 466/12 e foi aprovado por Comitê de Ética em Pesquisa de uma Universidade Pública em 10/09/2018, sob o parecer no 2.892.400. Responsáveis e crianças declararam seu aceite à participação por meio da assinatura dos Termos de Consentimento Livre e Esclarecido e de Assentimento, respectivamente.

\section{RESULTADOS}

Participaram da pesquisa 51 crianças, sendo $52,9 \%$ do sexo feminino. Predominou a idade de 10 anos (82,4\%), com média de idade 10,2 e desvio-padrão igual a 0,6 anos. Em relação ao IVC, com base nos três blocos de itens, as crianças avaliaram favoravelmente, considerando que o IVC geral e por blocos apresentou índices superiores a $90 \%$.

Mediante esses resultados, é possível afirmar que o caça-palavras foi validado semanticamente de forma positiva, atendendo ao que nele se buscou avaliar. Os pequenos ajustes na forma sugeridos por algumas crianças, a exemplo de aumento no tamanho de letras e/ou retirada de figuras ou de palavras consideradas de difícil compreensão, foram acatados pelos pesquisadores (Tabela 1).

A descrição dos depoimentos extraídos das entrevistas individuais com as crianças possibilitou a elaboração de duas nuvens de palavras, por meio do software IRaMuTeQ (Figura 2), de modo que cada questão respondida pelas crianças gerou 51 textos, os quais foram submetidos à análise pelo software. Dessa forma, os textos processados permitiram a visualização das respostas, evidenciando o conhecimento das crianças sobre a tuberculose e a perspectiva delas sobre a capacidade informativa da tecnologia.

A análise da primeira nuvem resultou em 56 segmentos de texto, com a identificação de 1.507 palavras, 243 palavras distintas e 104 hapax (palavras com frequência um). Destacaram-se as palavras: doença, tuberculose, entender, passar, pegar, tosse, outro, saliva e importante, todas elas em um contexto lexical relacionado à compreensão das crianças sobre a doença. $\mathrm{Na}$ segunda nuvem, obtiveram-se 54 segmentos de texto, com ocorrência de 243 palavras e 122 hapax, com destaque para as palavras: sim, tuberculose, doença, ajuda, entender, aprendere importante, mostrando que as crianças reconhecem a tecnologia como ferramenta para aprenderem sobre a doença. 
Tabela 1. Resultados dos Índices de Validade de Conteúdo, por blocos e geral, de acordo com as respostas das crianças. Belém, Pará, Brasil, 2019.

\begin{tabular}{llll}
\hline $\begin{array}{c}\text { Blocos de avaliação } \\
\text { do caça-palavras }\end{array}$ & \multicolumn{1}{c}{ Objetivos } & IVC* (\%) & $\begin{array}{c}\text { IVC* Geral } \\
\text { (\%) }\end{array}$ \\
\hline Impressão geral & $\begin{array}{l}\text { Identificar a impressão geral da criança sobre o conjunto de texto, } \\
\text { forma e capacidade de informação da tecnologia. }\end{array}$ & 98,3 \\
\hline Conteúdo do texto & $\begin{array}{l}\text { Identificar se, para a criança, as informações no texto estão claras e } \\
\text { são importantes para apreender conhecimentos sobre a doença. }\end{array}$ & 94,9 & 95,9 \\
\hline Forma da tecnologia & $\begin{array}{l}\text { Avaliar o grau de dificuldade da criança em solucionar o jogo de } \\
\text { caça-palavras e se sua forma e aparência são atraentes e facilitam o } \\
\text { aprendizado sobre a doença. }\end{array}$ & 96,6 \\
\hline
\end{tabular}

*IVC = Índice de Validade de Conteúdo

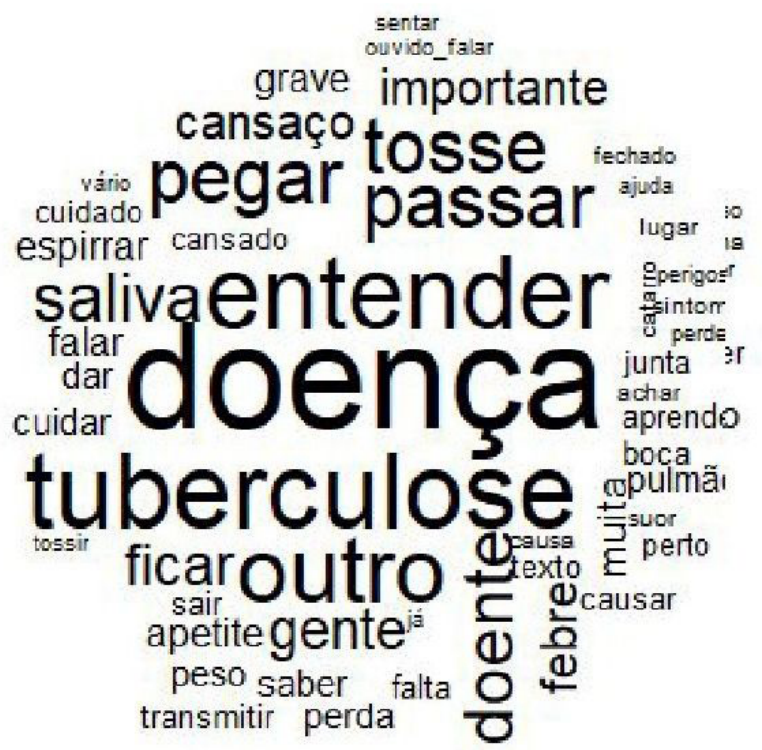

Figura 2. Nuvens de palavras relacionadas à compreensão dos conhecimentos sobre a tuberculose e a capacidade informativa da tecnologia educacional. Belém, Pará, Brasil, 2019.

Fonte: Elaborada pelos autores.

Na primeira nuvem, caracterizaram-se os aspectos referentes à compreensão sobre a tuberculose. As palavras selecionadas mostram claramente que as crianças conseguiram apreender as principais ideias sobre a doença no que concerne à transmissão, assim como aos sinais e sintomas. Na segunda, identifica-se, pelas palavras em destaque, que as crianças consideraram o caça-palavras uma ferramenta com potencial informativo para as pessoas, em geral, aprenderem e entenderem sobre a tuberculose.

\section{DISCUSSÃO}

A educação em saúde é uma importante ferramenta na promoção da saúde e na prevenção de doenças, principalmente para aquelas que têm relevância no contexto da saúde coletiva, a exemplo da tuberculose. A informação clara e precisa é primordial para prevenir sua expansão como problema de saúde

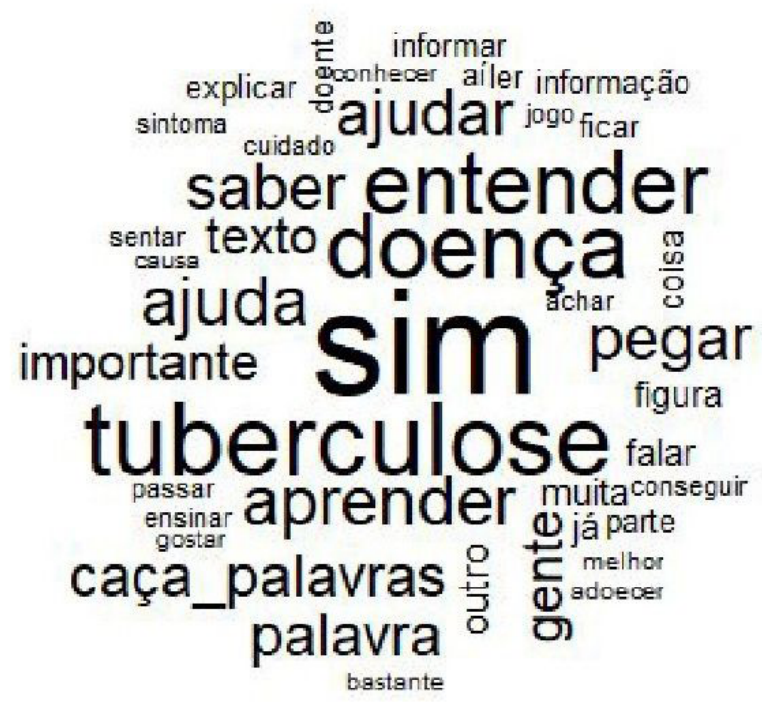

pública, posto que ela é o elemento facilitador da mobilização social em torno da doença, desde que conte com a participação ativa daqueles aos quais se destina e propicie a facilidade de comunicação. ${ }^{20}$

O caça-palavras foi considerado validado semanticamente, dado que os valores do IVC foram superiores a $90 \%$, tanto nos três blocos como no cômputo geral. Outro fator considerado foi sua validade no que se refere à clareza dos itens, facilidade na leitura e compreensão do texto, além de sua apresentação. Esses aspectos, na validação semântica, são relevantes, pois traduzem a avaliação daqueles que utilizarão a tecnologia. Ao mensurar esses aspectos, atende-se ao rigor científico exigido nos processos de validação. ${ }^{11,21,22}$

Quanto à tecnologia ser uma ferramenta que possibilite a compreensão sobre a tuberculose, a validação semântica mostrou a importância de se escolher cuidadosamente as palavras que 
permitam o entendimento compatível com o público leigo e faixa etária. Esse aspecto é importante, considerando-se que, na área da saúde, frequentemente divulgam-se as informações em linguagem técnica, que dificulta a compreensão pelo públicoalvo, em especial, ao tratar-se de grupos diferenciados, como as crianças ou pessoas com necessidades especiais. ${ }^{23,24}$

Ressalta-se a importância de atentar para a avaliação de materiais educativos em saúde, com relação à estrutura coesa, organizada, com linguagem adequada e suficiente para sua compreensão. É importante que o conteúdo mantenha o foco no tema e que seus textos apresentem sequência lógica de ideias, partindo de contextos mais gerais para assuntos mais específicos, ${ }^{24}$ o que foi considerado neste estudo ao tratar as questões sobre a tuberculose, partindo de seu conceito até chegar aos temas mais específicos referentes à transmissão, sinais e sintomas e como procurar ajuda e tratamento.

A necessidade de se levar informações válidas é fundamental, já que qualquer tecnologia e/ou instrumento elaborado e disponibilizado para intervenção em saúde, assim como para qualquer outro tipo de medida, deve dar voz ao público-alvo. Esse aspecto é reforçado, neste estudo, posto que foram consideradas crianças em idade escolar, fase de assimilação de conhecimentos e estímulo ao pensamento crítico e ao se produzir tecnologias educacionais de forma simples, clara e objetiva, que podem proporcionar maior conhecimento e adesão da criança e de sua família ao tema em questão. ${ }^{25,26}$

Para qualquer grupo, em qualquer faixa etária, deve-se considerar, de forma contextualizada, a quantidade e o tipo de informações que o público-alvo deseja ou precisa para se sentir informado e estimulado à mudança de práticas, não esquecendo que tais mudanças estão na dependência de experiências e informações provenientes de outras fontes. Materiais escritos em linguagem de fácil entendimento, a exemplo do caça-palavras, podem ampliar o desenvolvimento de habilidades para adesão às condutas de prevenção e tratamento de vários agravos. ${ }^{27}$

Nas escolas, práticas inovadoras e ativas de ensino e aprendizagem promovem o acesso à educação inclusiva, sendo modelo adequado para a participação das crianças em assuntos na área da saúde. ${ }^{28}$ Entende-se necessário considerar a integralidade como um aspecto essencial para o cuidado, que ocorre conforme se compreende a criança em sua totalidade, no contexto familiar e comunitário, considerando os aspectos que a formam como sujeitos ativos, respeitando-se as suas singularidades. ${ }^{29}$

Iniciativas globais têm incentivado práticas de estímulo ao desenvolvimento na infância, a partir de estruturas e atividades multissetoriais, ${ }^{30}$ que incluem, entre outras, ações de saúde, acesso a serviços, proteção e oportunidades de aprendizagem. ${ }^{31}$ Destaca-se a importância das intervenções individuais e comunitárias buscando ampliação do acesso aos cuidados, redução de custos e características salutares de empoderamento para mudanças sociais. Dessa forma, a articulação entre os profissionais, serviços de saúde e outros setores sociais, como escolas, é relevante à vigilância à saúde da criança. ${ }^{32,33}$
Assim, as questões relativas à saúde não devem ser consideradas apenas em suas características biológicas, e sim compreendidas como um processo e um produto do cotidiano, considerando sua determinação a partir dos aspectos socioculturais, políticos e econômicos relacionados às condições de vida e saúde da população. Esse debate é reconhecido e se fortalece no Brasil, reafirmando a escola como um importante espaço de favorecimento à vida com qualidade..$^{34}$

Dessa forma, entende-se que a educação em saúde pode acontecer em diferentes espaços, como nas escolas, propiciando atividades de orientação para promoção da saúde e prevenção de agravos importantes, a exemplo da tuberculose. Ressalta-se, assim, o papel do educador em saúde, aprimorando metodologias para o alcance de objetivos, valendo-se de tecnologias educativas validadas que sejam capazes de compartilhar informações seguras e adequadas ao público de interesse. ${ }^{35}$

\section{CONCLUSÃO E IMPLICAÇÕES PARA A PRÁTICA}

A pesquisa alcançou seu objetivo ao mostrar que a tecnologia permite esclarecer e informar de forma lúdica e tecnicamente correta sobre um tema importante como a tuberculose. Foi considerada adequada e poderá se constituir em ferramenta válida para utilização nas ações educativas em saúde por professores e/ou profissionais de saúde e intermediar a informação para a prevenção do adoecimento, fazendo circular informação precisa tanto para a comunidade escolar como para a comunidade em geral. Dar voz ao público-alvo incluindo os escolares no processo de validação, propiciou disponibilizar um material de fácil leitura, com linguagem acessível e grandes possibilidades para cumprir essa finalidade.

Como limitação da pesquisa, aponta-se sua realização exclusivamente com crianças estudantes em escola pública, pois as suas respostas e interesse pela tecnologia podem ser distintas daquelas crianças provenientes de escolas privadas Contudo, a tecnologia tem potencial para contribuir à prática de pesquisadores e profissionais da área de saúde e educação, pois constitui-se em material educativo que supera a prática de educação em saúde verticalizada e tradicional.

Entende-se, também, que as crianças poderão desempenhar importante papel como multiplicadores na divulgação de informações corretas sobre a tuberculose, a partir de sua compreensão sobre o tema facilitada pela tecnologia, principalmente quando ele é tratado de forma lúdica. Na sequência, para oportunizar novos estudos, há a proposta do teste do caça-palavras em relação ao Letramento em Saúde dos escolares, relacionado à identificação, prevenção e tratamento da tuberculose.

\section{FINANCIAMENTO}

O presente trabalho foi realizado com apoio da Coordenação de Aperfeiçoamento de Pessoal de Nível Superior - Brasil (CAPES) - Código de Financiamento 001. Programa Nacional de Cooperação Acadêmica - PROCAD/CAPES, edital nº 071/2013. 


\section{CONTRIBUIÇÕES DOS AUTORES}

Desenho do estudo. Ivaneide Leal Ataíde Rodrigues. Paula Daniella de Abreu. Claudia Benedita dos Santos. Laura Maria Vidal Nogueira. Eliane Maria Ribeiro de Vasconcelos. Marta Angélica lossi Silva.

Coleta ou produção dos dados. Ivaneide Leal Ataíde Rodrigues. Paula Daniella de Abreu. Claudia Benedita dos Santos. Laura Maria Vidal Nogueira.

Análise de dados. Ivaneide Leal Ataíde Rodrigues. Paula Daniella de Abreu. Claudia Benedita dos Santos. Alexandre Aguiar Pereira. Lucila Castanheira Nascimento.

Interpretação dos resultados. Ivaneide Leal Ataíde Rodrigues. Paula Daniella de Abreu. Claudia Benedita dos Santos. Alexandre Aguiar Pereira. Lucila Castanheira Nascimento. Eliane Maria Ribeiro de Vasconcelos. Marta Angélica lossi Silva.

Redação e revisão crítica do manuscrito. Ivaneide Leal Ataíde Rodrigues. Laura Maria Vidal Nogueira. Alexandre Aguiar Pereira. Paula Daniella de Abreu. Lucila Castanheira Nascimento. Eliane Maria Ribeiro de Vasconcelos. Marta Angélica lossi Silva. Claudia Benedita dos Santos.

Aprovação da versão final do artigo. Ivaneide Leal Ataíde Rodrigues. Laura Maria Vidal Nogueira. Alexandre Aguiar Pereira. Paula Daniella de Abreu. Lucila Castanheira Nascimento. Eliane Maria Ribeiro de Vasconcelos. Marta Angélica Iossi Silva. Claudia Benedita dos Santos.

Responsabilidade por todos os aspectos do conteúdo e a integridade do artigo publicado. Ivaneide Leal Ataíde Rodrigues. Laura Maria Vidal Nogueira, Alexandre Aguiar Pereira. Paula Daniella de Abreu. Lucila Castanheira Nascimento. Eliane Maria Ribeiro de Vasconcelos. Marta Angélica lossi Silva. Claudia Benedita dos Santos.

\section{EDITOR ASSOCIADO}

Candida Caniçali Primo

\section{REFERÊNCIAS}

1. World Health Organization. Global tuberculosis report 2019 [Internet]. Geneva:WHO; 2019 [citado 19 out 2020]. Disponível em: https://apps. who.int/iris/bitstream/handle/10665/329368/9789241565714-eng pdf?ua $=1$

2. Ministério da Saúde (BR), Secretaria de Vigilância em Saúde, Departamento de Vigilância Epidemiológica. Brasil livre da tuberculose: plano nacional pelo fim da tuberculose como problema de saúde pública [Internet]. Brasília (DF): Ministério da Saúde;2017 [citado 20 out 2020]. Disponível em: http://portalarquivos2.saude.gov.br/images/pdf/2017/ junho/29/plano_nacional_tb_web.pdf

3. Ministério da Saúde (BR), Secretaria de Vigilância em Saúde, Departamento de Vigilância Epidemiológica. Boletim epidemiológico tuberculose 2020 [Internet]. Brasília (DF): Ministério da Saúde; 2020. 40 p. [cited 2020 Oct 21]. Disponível em: https://antigo.saude.gov.br/ images/pdf/2020/marco/24/Boletim-tuberculose-2020-marcas--1-.pdf

4. Wanzeler WA, Rodrigues ILA, Nogueira LMV, Brasil GB, Santos CB. Álbum seriado sobre tuberculose para adolescentes. In: Teixeira $\mathrm{E}$, editor. Desenvolvimento de tecnologias cuidativo-educacionais. 2. ed. Porto Alegre (RS): Moriá; 2020. p. 133-143.

5. Xu T, Tomokawa S, Gregorio Jr ER, Mannava P, Nagai M, Sobel H School-based interventions to promote adolescent health: a systematic review in low- and middle-income countries of WHO Western Pacific Region. PLoS One. 2020 mar;15(3):e0230046. http://dx.doi.org/10.1371/ journal.pone.0230046.

6. Silva KVLG, Gonçalves GAA, Santos SB, Machado MFAS, Rebouças $\mathrm{CBA}$, Silva VM et al. Training of adolescent multipliers from the perspective of health promotion core competencies. Rev Bras Enferm. 2018 jan fev;71(1):89-96. http://dx.doi.org/10.1590/0034-7167-2016-0532.

7. Pueyo-Garrigues M, Whitehead D, Pardavila-Belio MI, Canga-Armayor A, Pueyo-Garrigues S, Canga-Armayor N. Health education: a Rogerian concept analysis. Int J Nurs Stud. 2019 jun;94:131-8. http://dx.doi. org/10.1016/j.jinurstu.2019.03.005.

8. Silva DML, Carreiro FA, Mello R. Educational technologies in nursing assistance in health education: integrating review. J Nurs UFPE on line. 2017 Feb;11(Suppl. 2):1044-51.

9. Nietsche EA, Lima MGR, Rodrigues MGS, Teixeira JA, Oliveira BNB Motta CA et al. Innovative technologies of nursing care. Rev Enferm UFSM [Internet]. 2012; [citado 2020 out 21];2(1):182-9. Disponível em: https://periodicos.ufsm.br/reufsm/article/view/3591/3144

10. Lima ACMACC, Bezerra KC, Sousa DMN, Vasconcelos CTM, Coutinho JFV, Oriá MOB. Educational technologies and practices for prevention of vertical HIV transmission. Rev Bras Enferm. 2018;71(Suppl. 4):175967. http://dx.doi.org/10.1590/0034-7167-2016-0333.

11. Alexandre NMC, Coluci MZO. Content validity in the development and adaptation processes of measurement instruments. Cien Saude Colet. 2011 Jul;16(7):3061-8. http://dx.doi.org/10.1590/S1413-81232011000800006.

12. Teixeira T, Nascimento MHM. Pesquisa metodológica: perspectivas operacionais e densidades participativas. In: Teixeira E, editor. Desenvolvimento de tecnologias cuidativo-educacionais. 2. ed. Porto Alegre: Moriá; 2020. p. 51-61.

13. Massaroli A, Martini JG, Lino MM, Spenassato D, Massaroli R. The Delphi Method as a methodological framework for research in nursing Texto Contexto Enferm. 2017 jan;26(4):e1110017. http://dx.doi. org/10.1590/0104-07072017001110017.

14. Pará, Secretaria de Estado de Saúde Pública, Coordenação Estadual do Programa de Controle da Tuberculose. Plano Estadual do Programa de Controle da Tuberculose 2018. Belém; 2018.

15. André SR, Nogueira LMV, Rodrigues ILA, Cunha TN, Palha PF, Santos $\mathrm{CB}$. Tuberculosis associated with the living conditions in an endemic municipality in the North of Brazil. Rev Latino-Am Enfermagem. 2020 ago;28:e3343. http://dx.doi.org/10.1590/1518-8345.3223.3343.

16. Souza MAR, Wall ML, Thuler ACMC, Lowen IMV, Peres AM. The use of IRAMUTEQ software for data analysis in qualitative research. Rev Esc Enferm USP. 2018 out;52:e03353. http://dx.doi.org/10.1590/s1980220x2017015003353.

17. Deon KC, Santos DMSS, Reis RA, Fegadolli C, Bullinger M, Santos CB. Translation and cultural adaptation of the brazilian version of DISABKIDS $®$ Atopic Dermatits Module (ADM). Rev Esc Enferm USP. 2011 abr;45(2):450-7. http://dx.doi.org/10.1590/S0080-62342011000200021.

18. Deon KC, Santos DMSS, Bullinger M, Santos CB. Preliminary psycometric assessment of the Brazilian version of the DISABKIDS $®$ Atopic Dermatitis Module. Rev Saude Publica. 2011 set;45(6):1072-8 http://dx.doi.org/10.1590/S0034-89102011005000067.

19. Fegadolli C, Reis RA, Martins STA, Bullinger M, Santos CB. Adaptation of the generic DISABKIDS $®$ module for Brazilian children and adolescents with chronic disorders. Rev Bras Saúde Mater Infant. $2010 \mathrm{jan} / \mathrm{mar} ; 10$ (1):95105. http://dx.doi.org/10.1590/S1519-38292010000100010.

20. Okeyo ILA, Dowse R. An illustrated booklet for reinforcing community health worker knowledge of tuberculosis and facilitating patient counselling. Afr J Prim Health Care Fam Med. 2018 maio; 10(1):a1687. http://dx.doi.org/10.4102/phcfm.v10i1.1687.

21. Brasil GB, Rodrigues ILA, Nogueira LMV, Palmeira IP. Educational technology for people living with HIV: validation study. Rev Bras Enferm. 2018;71(Suppl. 4):1657-62. http://dx.doi.org/10.1590/0034-7167-20170824.

22. Sousa EKS, Morais EJS, Amorim FCM, Oliveira ADS, Sousa KHJF, Almeida CAPL. Elaboration and validation of an educational technology related to violence against women. Esc Anna Nery. 2020 maio;24(4):e20190314 http://dx.doi.org/10.1590/2177-9465-ean-2019-0314. 


\section{Tecnologia educacional sobre tuberculose}

Rodrigues ILA, Nogueira LMV, Pereira AA, Abreu PD, Nascimento LC, Vasconcelos EMR, Silva MAI, Santos CB

23. Kronish IM, Goldfinger JZ, Negron R, Fei K, Tuhrim S, Arniella G et al The effect of peer education on stroke prevention: the prevent recurrence of all inner-city strokes through education (PRAISE) randomized controlled trial. Stroke.2014 set;45(11):3330-6. http://dx.doi.org/10.1161/ STROKEAHA.114.006623.

24. Galindo-Neto NM, Alexandre ACS, Barros LM, Sá GGM, Carvalho $\mathrm{KM}$, Caetano JA. Creation and validation of an educational video for deaf people about cardiopulmonary resuscitation. Rev. Latino-Am. Enfermagem. 2019 mar;27:e3130. http://dx.doi.org/10.1590/15188345.2765.3130.

25. Ernst G, Menrath I, Lange K, Eisemann N, Staab D, Thyen U et al Development and evaluation of a generic education program for chronic diseases in childhood. Patient Educ Couns. 2017 jun;100(6):1153-60 http://dx.doi.org/10.1016/j.pec.2017.01.001.

26. Romeiro V, Bullinger M, Marziale MHP, Fegadolli C, Reis RA, Silveira RCCP et al. DISABKIDS ${ }^{\circledR}$ in Brazil: advances and future perspectives for the production of scientific knowledge. Rev Latino-Am Enfermagem 2020 abr;28:e3257. http://dx.doi.org/10.1590/1518-8345.3003.3257.

27. Wild CFW, Nietsche E, Salbego C, Teixeira E, Favero NB. Validation of educational booklet: an educational technology in dengue prevention. Rev Bras Enferm. 2019 set;72(5):1318-25. http://dx.doi.org/10.1590/00347167-2018-0771.

28. Koller D. 'Kids need to talk too': inclusive practices for children's healthcare education and participation. J Clin Nurs. 2017 set;26(17-18):2657-68. http://dx.doi.org/10.1111/jocn.13703.
29. Furtado MCC, Mello DF, Pina JC, Vicente JB, Lima PR, Rezende VD. Nurses' actions and articulations in child care in primary health care. Texto Contexto Enferm. 2017 mar;27(1):e0930016. http://dx.doi. org/10.1590/0104-07072018000930016.

30. Britto PR, Lye SJ, Proulx K, Yousafzai AK, Matthews SG, Vaivada T et al Nurturing care: promoting early childhood development. Lancet. 2017 jan;389(10064):91-102. http://dx.doi.org/10.1016/S0140-6736(16)313903.

31. Black MM, Walker SP, Fernald LCH, Andersen CT, Digirolamo AM, Lu $\mathrm{C}$ et al. Early childhood development coming of age: science through the life course. Lancet. 2017 jan;389(10064):77-90. http://dx.doi org/10.1016/S0140-6736(16)31389-7.

32. Grant J, Lines L, Darbyshire P, Parry Y. How do nurse practitioners work in primary health care settings? A scoping review. Int J Nurs Stud. 2017 jun;75(4):51-7. http://dx.doi.org/10.1016/j.jinurstu.2017.06.011.

33. Yakuwa MS, Neill S, Mello DF. Nursing strategies for child health surveillance. Rev Latino-Am Enfermagem. 2018;26:e3007. http://dx.doi. org/10.1590/1518-8345.2434.3007.

34. Silva CS, Bodstein RCA. A theoretical framework on intersectoral practice in School Health Promotion. Cien Saude Colet. 2016 Jun;21(6):1777-88. http://dx.doi.org/10.1590/1413-81232015216.08522016.

35. Halse KM, Fonn M, Christiansen B. Health education and the pedagogica role of the nurse: Nursing students learning in the clinical setting. $J$ Nurs Educ Pract. 2014 out;4(3):30-7. http://dx.doi.org/10.5430/jnep. v4n3p30. 